\title{
Transient hypoxia following nitrogen spray cryotherapy for Barrett's Esophagus: A case report
}

\author{
Matthew L Ritz, Andrew Murray and Andrew Gorlin* \\ Department of Anesthesiology, Mayo Clinic Hospital - Phoenix, Arizona, USA
}

\begin{abstract}
We present here a case of intermittent hypoxia during nitrogen spray cryotherapy for the treatment of Barrett's Esophagus. Hypoxia has been previously described as a complication following liquid nitrogen cryotherapy for the treatment of malignant airway disease using bronchoscopy, but to our knowledge it has not been reported during endoscopy. Knowing this potential complication, endoscopy involving nitrogen spray cryotherapy may warrant additional airway interventions, including intubation, in select patients.
\end{abstract}

\section{Introduction}

Hypoxia is a described complication following liquid nitrogen cryotherapy for the treatment of malignant airway disease using bronchoscopy [1]. Liquid nitrogen cryotherapy has been shown to be an effective therapy for the treatment of Barrett's Esophagus [2]. We present here a case of intermittent hypoxia during nitrogen spray cryotherapy for the treatment of Barrett's Esophagus.

\section{Case presentation}

The patient was a 70-year-old male with a past medical history significant for gastro esophageal reflux disease, Barrett's Esophagus, and Class III obesity with a body mass index greater than 40 . The patient had undergone previous radio frequency ablation for treatment of Barrett's Esophagus. The patient presented for a surveillance endoscopy and nitrogen cryotherapy. A nasal cannula was placed with side stream capnography, and intravenous induction of anesthesia was performed with propofol and lidocaine. A propofol infusion was used for maintenance of anesthesia and titrated appropriately to maintain depth of anesthesia, the patient was breathing spontaneously for the duration of the case. The endoscope was introduced without complication. Nitrogen spray cryotherapy was performed for twenty seconds for two cycles at each treatment site. A total of four different sites were ablated. Ventilation tubing was inserted adjacent to the endoscope and suction aided ventilation of gases was performed through the ventilation tubing during, and for 20 seconds after the ablation procedure. The patient developed hypoxia with desaturation into the 80 s by pulse oximetry during each ablation. The hypoxia resolved each time ablation was completed. The procedure was ultimately completed successfully and the patient was taken to the PACU with a nasal cannula in place which was ultimately discontinued, he was discharged without further episodes of hypoxia.

\section{Discussion}

Nitrogen spray cryotherapy (SCT) for Barrett's Esophagus is performed via a spray catheter that is inserted through an endoscopeworking channel to direct low-pressure liquid nitrogen at areas that require ablation. The catheter tip is positioned 1 to $2 \mathrm{~cm}$ away from the target mucosa, and liquid nitrogen is applied until ice becomes visible. Freezing occurs to negative 196 degrees Fahrenheit. The non-contact function of SCT introduces formidable challenges for management of the airway. Notably, in the endoscopic application, the rapid expansion of liquid nitrogen requires continuous decompression via a $5-\mathrm{mm}$ diameter oral-gastric tube to prevent downstream intestinal perforation [3].

Hypoxia can occur due to rapid expansion of nitrogen displacing inspired oxygen; this phenomenon has been more frequently described during bronchoscopy and SCT for malignant airway disease. This hypoxia can be profound, and it persists until oxygen is reintroduced and the high concentration of nitrogen gas is washed out of the lungs. This can be partially attenuated by performing preoxygenation with 100 percent oxygen prior to ablation. Barotrauma has also been documented due to the rapidly climbing pressure within the airway [4]. Each 1 $\mathrm{mL}$ of liquid nitrogen expands to approximately $700 \mathrm{~mL}$ of nitrogen gas in the endoluminal space [5]. Unlike SCT via an endoscope, it is impractical and potentially detrimental to have a suction catheter in the airway, thus it is critical to ensure that passive venting can occur around the bronchoscope or endotracheal tube. In the airways, active suctioning is generally not feasible because active suctioning of air from the lungs can reduce oxygen content available for respiration and could collapse portions of lung. This ultimately reduces the airway caliber available for passive venting of the newly formed nitrogen gas. Also, any additional tubing in the airway besides the bronchoscope and/or intubating modality decreases the available working space to treat the target and passively vent gases. Currently, the recommendation for ensuring safe, passive egress of the nitrogen gas produced by SCT is a $8.5 \mathrm{~mm}$ endotracheal tube of a standard adult rigid bronchoscope (usually inner diameter of $9 \mathrm{~mm}$ or greater) [1]. Rare instances of pneumothorax, pneumomediastinum, and nitrogen gas embolism have been reported during bronchoscopy and SCT. SCT is particularly

*Correspondence to: Andrew Gorlin, Department of Anesthesiology, Mayo Clinic Hospital - Phoenix, Arizona, USA, E-mail: gorlin.andrew@mayo.edu

Received: July 21, 2020; Accepted: August 07, 2020; Published: August 11, 2020 
advantageous for both endoscopy and bronchoscopy as there is no heat delivery or ignition source, thus there is no risk of combustion. It is therefore possible to administer high-inspired concentrations of oxygen during these procedures. Compared to thermal or mechanical modalities, the procedure is relatively painless and may actually have an analgesic effect on nerve fibers. In addition, due to its vasoconstrictive effect, it is beneficial in achieving hemostasis [6].

\section{Conclusion}

In our patient, we observed intermittent hypoxia during SCT that was fortunately well tolerated. Given the hypoxia that can be observed while using SCT via an endoscope, it would be reasonable to consider intubating patients undergoing these procedure as well as performing aggressive preoxygenation prior to each cycle of ablation; especially if intermittent hypoxia would be poorly tolerated.

\section{Conflicts of interests}

None.

\section{Financial disclosures}

None.

\section{Patient consent}

Written consent has been obtained from the patient to publish this case report.

\section{References}

1. Browning R, Turner JF, Parrish S (2015) Spray cryotherapy (SCT): institutional evolution of techniques and clinical practice from early experience in the treatment of malignant airway disease. $J$ Thorac Dis 7: S405-S414.

2. Visrodia K, Zakko L, Wang KK (2018) Mucosal ablation in patients with Barrett's Esophagus: Fry or Freeze? Dig Dis Sci 63: 2129-2135.

3. Dibardino DM, Lanfranco AR, Haas AR (2016) Bronchoscopic Cryotherapy. Clinical applications of the cryoprobe, cryospray, and cryoadhesion. Ann Am Thorac Soc 13: 1405-1415.

4. Finley DJ, Dycoco J, Sarkar S, Krimsky WS, Sherwood JT, et al. (2012) Airway spray cryotherapy: Initial outcomes from a multiinstitutional registry. Ann Thorac Surg 94 : 199-203.

5. Epstein J, Krochmal R, Hannallah M (2018) Precipitous drop in hemoglobin oxygen saturation during spray liquid nitrogen cryotherapy of tracheobronchial lesions. $J$ Cardiothorac Vasc Anesth 32: e68-e69.

6. Moore RF, Lile DJ, Abbas AE (2017) Current status of spray cryotherapy for airway disease. J Thorac Dis 9: S122-S129.

Copyright: (C2020 Ritz ML. This is an open-access article distributed under the terms of the Creative Commons Attribution License, which permits unrestricted use, distribution, and reproduction in any medium, provided the original author and source are credited. 